\title{
Erratum: Motional Averaging of Nuclear Resonance in a Field Gradient [Phys. Rev. Lett. 114, 197601 (2015)]
}

Nanette N. Jarenwattananon and Louis-S. Bouchard

(Received 12 May 2016; published 27 May 2016)

DOI: 10.1103/PhysRevLett.116.219903

In our Letter, Eq. (14),

$$
\kappa(T)=\frac{k T\left(-m \zeta_{-}^{2} \zeta_{+}-m \zeta_{-} \zeta_{+}^{2}+\zeta_{-} \gamma+\zeta_{-} \zeta_{+} \gamma+\zeta_{+}^{2} \gamma\right)}{m M \zeta_{-}^{3} \zeta_{+}^{3}},
$$

contains a typographical error. It should instead read

$$
\kappa(T)=\frac{k T\left(-m \zeta_{-}^{2} \zeta_{+}-m \zeta_{-} \zeta_{+}^{2}+\zeta_{-}^{2} \gamma+\zeta_{-} \zeta_{+} \gamma+\zeta_{+}^{2} \gamma\right)}{m M \zeta_{-}^{3} \zeta_{+}^{3}} ;
$$

i.e., the third term inside the parenthesis should be $\zeta_{-}^{2} \gamma$ (the square was missing). Our analysis of the temperature dependence did use the correct term with the square. This typo does not affect any other equations and the conclusion of the Letter remains the same.

We thank Dr. Vladimír Lisý and Dr. Jana Tóthová for bringing this typo to our attention. 